\title{
Study on Anti - Multiple Access Interference in Satellite Communication of GNSS Ground Station
}

\author{
Siyuan Liang \\ Shaanxi Key Laboratory of Information Communication Network and Security, Xi'an University of Posts and \\ Telecommunications, Xi'an 710121, China. \\ telestorm@163.com
}

\begin{abstract}
In the global navigation satellite system (GNSS), communication between ground monitoring stations and master control stations can be easily disturbed by the multi-access interference (MAI). This paper proposes a Kalan filtering multiuser detector method to anti MAI that can improve receiver bit error rate (BER). This method is verified by simulation, the results shows MAI suppression obviously.
\end{abstract}

Key words: GNSS, Kalan Filtering, Anti Multi-Access Interference.

\section{INTRODUCTION}

GNSS consists of satellite system, ground system and the user's system, among them monitoring stations and master control stations are important components of ground system of satellite navigation system [1]. As communication between ground monitoring stations and master control stations (CBMC) can be easily disturbed by the MAI, thus the study on the interference of monitoring station communication has becomes one of the most important keys in the satellite navigation area, the Fig. 1 is as follow.

If satellite communication link between master stations and monitoring stations are affected by interference, it will influence GNSS satellite orbit determination, ephemeris and clock correction calculation, make the GNSS accuracy and reliability reduce. [4] Therefore, the communication anti-interference technology in CBMC is one of the key technologies in the construction of satellite navigation system.

The uplink and downlink of CBMC includes the digital signal processing, radiofrequency signal processing and amplifier. The digital signal processing of CBMC shows as following Fig. 2.

The spread spectrum technology is used between monitoring stations and master control stations and PN sequence is chosen as spread spectrum code. The uplink digital signal processing is the first to perform cyclic redundancy check (CRC) and then to perform forward error correction coding (FEC). FEC coding type chooses low density parity check (LDPC) code with strong error correction performance. 


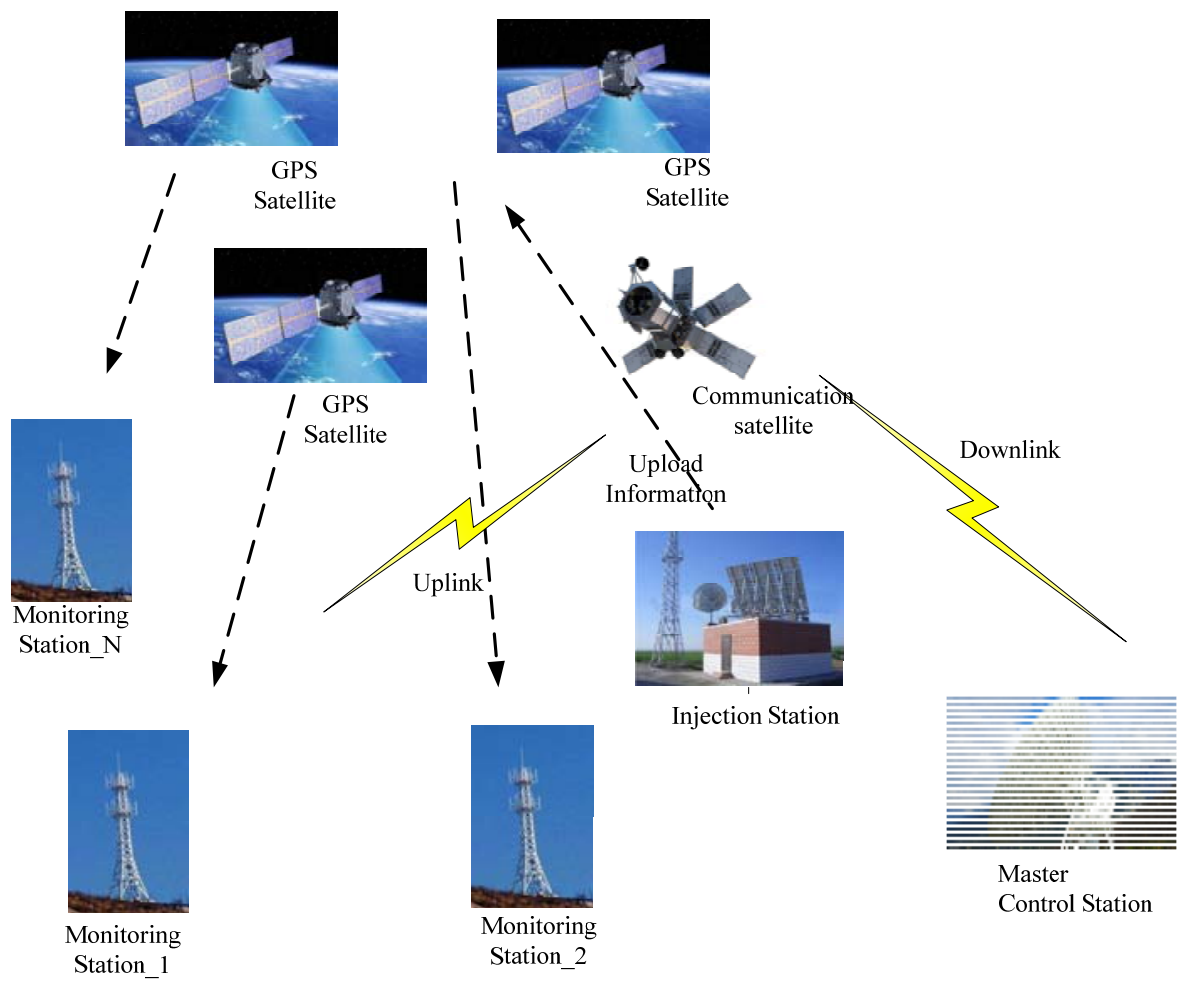

FIGURE 1. CBMC of GNSS.
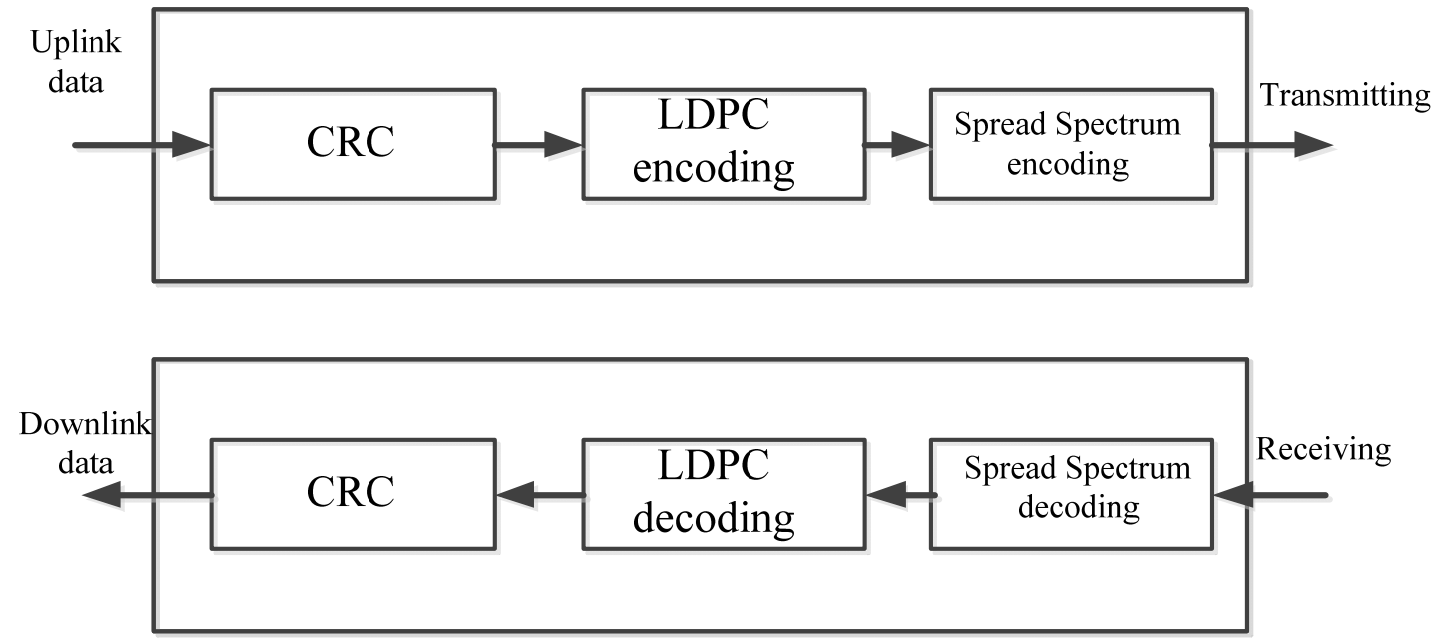

FIGURE 2. CBMC digital signal processing.

MATHEMATICAL MODELS

Defining

$$
\begin{gathered}
s_{i}=\left[s_{i}(0), s_{i}(1), s_{i}(2) \ldots s_{i}(N-1)\right] \\
y=[r(0), r(1), r(2) \ldots r(N-1)]
\end{gathered}
$$




$$
v=[n(0), n(1), n(2) \ldots n(N-1)]
$$

After demodulation, the up/downlink receiving signal express in vector form, as equation (4).

$$
y(k)=\sum_{i=1}^{M} A_{i}(k) b_{i}(k) s_{i}(k)+v(k)
$$

The multiuser detector for satellite q can be used to anti MAI, the output is given by equation (5).

$$
\hat{b}_{q}(k)=\operatorname{sgn}\left(<c_{q}(k), y(k)>\right)=\operatorname{sgn}\left(c_{q}^{T}(k) y(k)\right)
$$

In the equation (5), the tap-weight vector $c_{q}(k)$ characterizes the multiuser detector. In the following, we consider how to establish $c_{q}(k)$ for blind adaptive MAI multiuser detector. The $c_{q}(k)$ can be expressed as equation (6).

$$
c_{q}(k)=s_{q}-C_{q, \text { null }} \bullet w_{q}(k)
$$

Where, the $C_{q, \text { null }}$ is a $N \times(N-1)$ matrix, that is the null space of $s_{q}$. The $C_{q}(k)$ can adaptively change with weight vector $w_{q}(k) .[2,3]$.

\section{ANTI-MAI BASED ON KALMAN FILTERING}

An adaptive multiuser detection based on Kalan Filtering is designed to anti-MAI in master control station. The state equation and measurement equation are foundations of Kalan Filtering designing, which constitute the dynamic system model of multiuser detection.

Defining:

$$
z_{q}(k)=s_{q}^{T} y(k), d^{T}(k)=y^{T}(k) C_{q, n u l l}
$$

If $w_{q}(t)$ achieves $w_{q, o p t}(k)$, the measurement equation as following (8).

$$
z_{q}(k)=d(k) w_{q, o p t}(k)+e_{q}(k)
$$

The Kalan filtering equations as following:

The state estimation equation:

$$
w_{q, o p t}(k)=w_{q, o p t}(k-1)+g(k)\left[z(k)-d^{T}(k) w_{q, o p t}(k-1)\right]
$$

The gain equation:

$$
g(k)=K(k \mid k-1) d(k)\left[d^{T}(k) K(k \mid k-1) d(k)+\xi_{m m}\right]^{-1}
$$

The MSE estimation equation:

$$
K(k+1 \mid k)=K(k \mid k-1)-g(k) d^{T}(k) K(k \mid k-1)
$$

The tap-weight vector equation: 


$$
c_{q}(k)=s_{q}-C_{q, \text { null }} \bullet w_{q, o p t}(k)
$$

Where, $g(k)$ is gain of Kalan filtering, $K(k+1 \mid k)$ is MSE matrix and $K(1 \mid 0)=I$. In equation (10), is the minimum mean output energy of the dynamical system of satellite q, we can directly take $\xi_{m m}(k)=A_{q}(k)$.

\section{SIMULATION AND RESULTS}

The Mat lab simulation software is used to simulate the anti-MAI of the CBMC, and the number of users is 1 to 25. Spread frequency code adopts PN sequence, the length of it is 200 bits. The code gain of LDPC is $4 \mathrm{~dB}$. The result of simulation is as following Fig. 3.

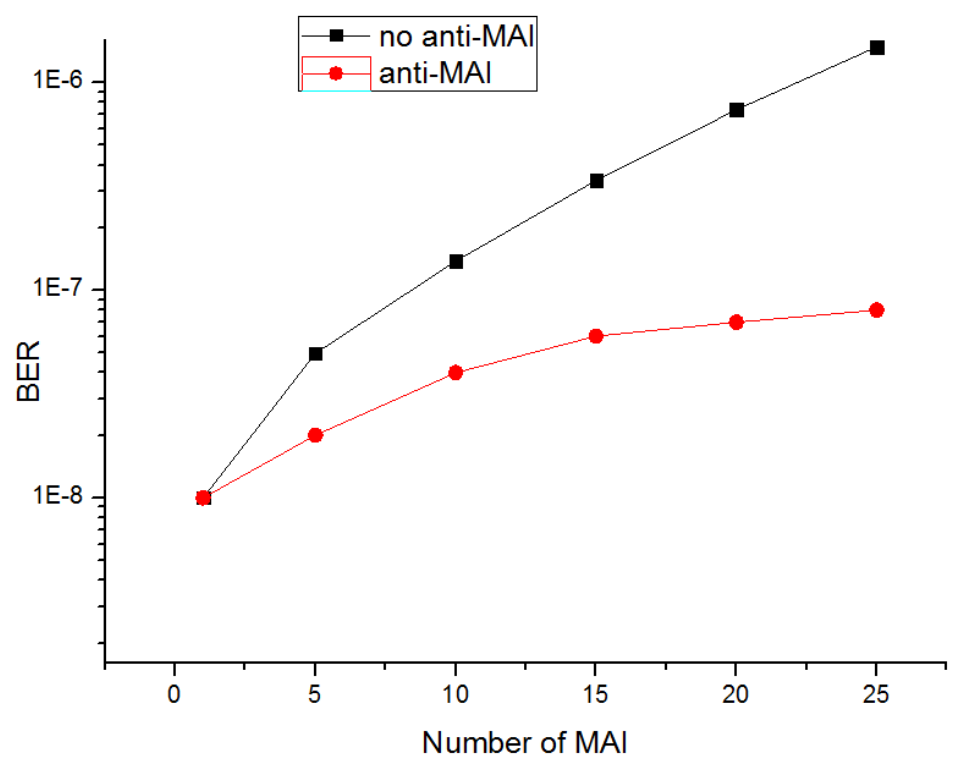

FIGURE 3. Simulation results.

According to Fig. 3, when using 200bit long PN sequence as spread frequency code, when the number of MAI is 5 the no anti-MAI and anti-MAI BER is about 10-8, and with increasing of number of MAI the error bit in no antiMAI condition increasing speed faster than anti-MAI condition. When the number of MAI is 15 the no anti-MAI BER is about 10-7 and anti-MAI BER is about 10-8. The Kalan filtering based anti-MAI method is useful that is verified by simulation.

\section{CONCLUSION}

The anti-MAI method can take CBMC downlink receiver stronger signal acquisition ability to enhance the satellite communication usability. In this paper, a Kalan filtering-based method is proposed to anti-MAI that can improve receiver BER. The method availability is proved by simulation results.

\section{ACKNOWLEDGMENTS}

This work was supported by Scientific Research Plan Projects of Shaanxi Education Department (No. 17JK0702) and National Natural Science Foundation of China under Grant 61371087. 


\section{REFERENCES}

1. R. Malawian, N. R. Moloisane, L. Nair, B.T. Maharaja, and U. A. K. Chide-Okonkwo, "Design and implementation of a wireless OBD II fleet management system," IEEE Sensors J., Vol. 17 (2017) No. 4, p. 1154-1164.

2. Xian-Da Zhang, Wei, Blind Adaptive Multiuser Detection Based on Kalan Filtering, IEEE Transactions on Signal Processing, Vol. 50(2002) No.1, p.87-95.

3. Y. Wang, C. D. Zhang, X. G. Hu, and Y. Z. Song, et al., "Robust Kalan filter based on different satellite types and its application in GPS/BDS precise point positioning," Journal of Chinese Inertial Technology, Vol. 24(2016) No. 6, p. 769-779. 